\title{
Artículos
}

\section{Control de la contaminación atmosférica en la Zona Metropolitana del Valle de México}

\section{Air pollution control in the Valley of Mexico Metropolitan Area}

\author{
Alejandro Navarro Arredondo*
}

\section{Resumen}

Este artículo analiza los mecanismos de control de la contaminación atmosférica en la Zona Metropolitana del Valle de México (ZMVM). Por esa razón, se utiliza el ciclo de políticas públicas para examinar el Programa para Mejorar la Calidad del Aire (Proaire), en términos de las medidas y acciones que propone. También se identifican las limitaciones en su diseño e implementación. Finalmente, se presentan las conclusiones del análisis.

Palabras clave: contaminación atmosférica; calidad del aire; Valle de México; política ambiental; programas urbanos; zona metropolitana.

\begin{abstract}
This article analyzes the air pollution control mechanisms at the Valley of Mexico Metropolitan Zone (ZMVM). For that reason, the public policy cycle is used to examine the Program to Improve Air Quality (Proaire), in terms of its proposed measures and actions. Afterward, the limitations in its design and implementation are identified. Finally, the conclusions of the analysis are presented.
\end{abstract}

Keywords: air pollution; air quality; Valley of Mexico; environmental policy; urban programs; metropolitan area.

* Universidad Autónoma Metropolitana, Unidad Xochimilco, División de Ciencias Sociales y Humanidades, Departamento de Política y Cultura. Dirección postal: Calz. del Hueso 1100, Villa Quietud, 04960, Coyoacán, Ciudad de México, México. Correo electrónico: galex. navarro@gmail.com 


\section{Introducción}

La contaminación atmosférica es un problema que tiene efectos adversos sobre la salud humana y el medio ambiente. Además, no es raro que las corrientes de aire puedan trasladar los contaminantes a través de diferentes territorios. Por lo tanto, este problema puede tener un alcance geográfico amplio y convertirse en un asunto regional. Al respecto, se ha recurrido a diferentes alternativas de solución para hacer frente a esta problemática. En ese sentido, la estrategia más usada son los programas urbanos de gestión de la calidad del aire (PUGCA).

Por lo anterior, el objetivo de este artículo es analizar los mecanismos de control de la contaminación atmosférica contemplados en el PUGCA de la ZMVM. Para ello, se utilizará el ciclo de políticas públicas como marco de análisis para examinar algunos aspectos del diseño y la implementación del Programa para Mejorar la Calidad del Aire (Proaire) (CAM, 2013). Así, este artículo se organiza en las siguientes secciones: $a$ ) la revisión del marco teórico del ciclo de políticas públicas; $b$ ) la caracterización del PUGCA de la ZMVM, en términos de las medidas y acciones que propone; c) la identificación de las limitaciones en su diseño e implementación; y d) las conclusiones del análisis.

\section{El proceso de las políticas públicas como marco de análisis del problema de la contaminación atmosférica}

La contaminación atmosférica es considerada uno de los problemas más apremiantes de la actualidad. De acuerdo con Kelly y Fussell (2015), el problema es tan grave que ha puesto en riesgo la sostenibilidad de los entornos urbanos. De hecho, en muchas ciudades, como Río de Janeiro, Shanghai, Beijing, Mumbai, Delhi, Yakarta, Ciudad de México y Manila, el nivel de contaminantes en la atmósfera es varias veces más alto que el límite permitido para proteger la salud humana. Por ello, hay quienes ubican a la contaminación atmosférica en la misma categoría de importancia de otros problemas como la lluvia ácida, el agotamiento de la capa de ozono o el calentamiento global (Kelly y Fussell, 2015).

Según Wang, Hawkins, Lebredo y Berman (2012), la gravedad de este problema se atribuye a la naturaleza compleja y multifactorial de las actividades generadoras de contaminantes, pero también a la ineficacia de las autoridades. Más aún, la ineficacia gubernamental se relaciona con la escasa información y comprensión del problema, así como con la falta de capacidad 
de los organismos encargados de atenderlo (Wolfram, 2016). Por ello, una pregunta básica que se desprende de esta perspectiva es, ¿qué puede permitir a las autoridades atender con mayor eficacia el problema de la contaminación atmosférica? Algunos consideran que el proceso de las políticas públicas puede proveer tanto los elementos teóricos como las herramientas prácticas para encontrar respuestas a esta interrogante (Campbell, 2010).

La idea del proceso (o ciclo) de las políticas públicas fue propuesta por primera vez por Lasswell (1956). Para ello, introdujo un modelo del proceso dividido en siete etapas: inteligencia, promoción, prescripción, invocación, aplicación, finalización y evaluación. Al hacer esto (Lasswell, 1971, p. 28), elaboró un "mapa conceptual para obtener una imagen generalizada de las fases principales de cualquier proceso de políticas públicas". Este número de etapas ha sido cuestionado y criticado, pero el modelo en sí mismo continúa teniendo éxito como marco de análisis de las políticas públicas. Durante las décadas de 1960 y 1970 se desarrollaron varias tipologías de dicho proceso; pero hoy, el modelo más convencional para describir una secuencia de políticas públicas está compuesto por las siguientes etapas: configuración de la agenda, formulación de soluciones, implementación y evaluación (Jann y Wegrich, 2007).

El ciclo de políticas públicas fue diseñado como un modelo de resolución de problemas, por lo que será nuestra guía para analizar la formulación de soluciones al problema de la contaminación atmosférica. Ahora bien, antes de comenzar dicho análisis es necesario señalar que un ciclo de políticas va más allá de las organizaciones públicas involucradas: no es un proceso exclusivo de las instancias gubernamentales. Un proceso de políticas públicas implica múltiples y diferentes organizaciones y/o individuos. Por lo tanto, este proceso se caracteriza por la presencia de varios intereses que pueden entrar en conflicto y por ello se genera un espacio donde dichos intereses interactúan: la agenda pública.

\section{Configuración de la agenda}

De acuerdo con Dunn (2004), el punto inicial de cualquier proceso de política pública es la intención de resolver un problema público; sin embargo, éste raramente se presenta como una cuestión aislada de otros fenómenos de la vida social. Hay muchas situaciones problemáticas en la sociedad, pero sólo son consideradas aquellas que coinciden con las visiones, aspiraciones e intereses de los actores de poder, quienes las impulsan e incorporan a la agenda pública. De esta forma, la agenda pública hace referencia al listado de pro- 
blemas a los que las autoridades prestarán atención en cierto momento. Ello lleva a preguntarse, ¿por qué las autoridades abordan un problema y no otro?, ¿por qué una iniciativa se transforma en política pública y otras no?

La respuesta es que la capacidad, el tiempo, los recursos o el margen de maniobra de las autoridades son limitados; por ello no podrán abordar todos los asuntos y prestarán atención sólo a algunos de ellos. En ese sentido, existen varios problemas compitiendo por capturar la atención gubernamental y se da un proceso en el que los interesados por visibilizar esos asuntos realizan esfuerzos conscientes y competitivos para incorporarlos a la agenda pública (Dutton, 1986). Se reconoce entonces que algunos problemas son más llamativos que otros, es decir, existen factores que aumentan la atención a ciertos asuntos; entre ellos están los siguientes:

- Valores. Los valores son la esencia de la formulación de las políticas. Inducen preferencias, prioridades, juicios y justifican acciones. Tienen varios orígenes: ideología, cultura, religión, creencias, conocimiento, etc. Es poco probable que el acceso de un problema a la agenda pública pueda ser legitimado sin hacer referencia a un conjunto de valores.

- Usos y costumbres. Las instituciones políticas tienen su propia inercia organizacional. El proceso de formulación de políticas se caracteriza por procedimientos y patrones a menudo enraizados en la cultura y la historia, que restringen o aceleran el acceso de un problema a la agenda pública. Varias veces tales restricciones aparecen bajo la forma de leyes o normas, pero igualmente pueden aparecer como procesos de legitimación socialmente construidos.

- Cabilderos y grupos de presión. Cualquier proceso de formulación de políticas moviliza grupos de presión, cabilderos informales u organizados, así como la opinión de expertos. Tales partes interesadas no siempre son visibles, pero tienen una influencia importante, pues permiten que ciertas preocupaciones e intereses encuentren un lugar dentro de la agenda de gobierno.

- Contingencias. El proceso de configuración de la agenda puede ser afectado por imprevistos o situaciones percibidas como asuntos de emergencia: crisis políticas, económicas, sociales o ambientales que facilitan que cierto problema se inserte de inmediato en la agenda pública.

En casi todo el mundo, la contaminación atmosférica es un problema que recibe cada vez más atención por parte de investigadores, autoridades y 
público en general. Para algunos, su inclusión en la agenda pública ha ocurrido gracias a la influencia de expertos y grupos de presión, así como por considerarse una situación de emergencia. En particular, la mala calidad del aire es un problema de salud pública mundial (Kelly y Fussell, 2015). Además, la percepción de emergencia sobre este problema se nutre de las aportaciones de organismos internacionales, como la Organización Mundial de la Salud (OMS), que ha estimado que el aumento de la contaminación atmosférica ha causado más de siete millones de muertes por año. Recientemente, esa organización la consideró carcinogénica para los seres humanos (OMS, 2014).

Una vez que el problema de contaminación atmosférica se ha colocado en la agenda pública y de gobierno, corresponde a las autoridades emprender acciones encaminadas a su solución. Sin embargo, la formulación de soluciones implica que varias partes interesadas lleven diferentes tipos de conocimiento (de grupos sociales, ciudadanos, actores económicos); experiencia (de personal técnico, científicos, expertos); y juicios (de la opinión pública, órganos electos, comités), para que todos estos elementos se integren al diseño de políticas públicas. Precisamente, esa situación da pie a la siguiente fase del proceso: la formulación de soluciones.

\section{Formulación de soluciones}

Según Dunn (2004), la formulación de soluciones (también conocida como diseño de alternativas) hace referencia a la etapa en la cual las políticas públicas son delineadas, a través de un análisis técnico, con el fin de alcanzar una meta. Así, luego de identificarse un problema, se deduce, estructura o diseña una intervención para resolverlo o, al menos, mitigarlo. Ello supone que el problema es descompuesto en sus elementos constitutivos para identificar sus características, causas, interconexiones y consecuencias. A partir de esa información se diseñan las políticas públicas. Al respecto, Jacobson (2002) considera que se han empleado, al menos, tres diferentes tipos de estrategias para reducir la contaminación atmosférica: reglas tipo command and control, incentivos económicos y programas urbanos de gestión de la calidad del aire.

Las reglas tipo command and control se pueden definir como la regulación directa de una industria o una actividad mediante una norma que establece lo que está permitido y lo que es ilegal. Esta regulación abarca una variedad de métodos que tratan de influir en la conducta de las empresas e individuos: leyes, incentivos y contratos. El componente de command se 
refiere a las normas o estándares que elabora la autoridad y que las industrias o los actores regulados deben cumplir forzosamente. La parte de control se refiere a las sanciones negativas que pueden resultar del incumplimiento de dichas normas o estándares: una sanción económica o el encarcelamiento (Jacobson, 2002).

En el caso de la regulación ambiental, el enfoque de command and control depende en gran medida del uso de estándares para garantizar mejoras en el medio ambiente. Estas normas rigen en qué medida una jurisdicción en particular necesita reducir sus emisiones, por lo que esos estándares requieren cierta comprensión de los efectos de la contaminación sobre la salud y el medio ambiente. Este enfoque se usa por varias razones, pero la principal es porque logra regular la contaminación gracias a la amenaza de la aplicación de la ley. Así, con dicho enfoque es más rápido persuadir a empresas e individuos que no cumplen con los estándares establecidos. También tiene beneficios políticos para el gobierno ya que la ciudadanía percibe que éste actúa de manera rápida y decisiva (Jacobson, 2002).

Sin embargo, el enfoque de command and control está lejos de ser una forma de control de la contaminación atmosférica libre de problemas. Desde hace tiempo ha recibido varias críticas. Una de ellas se refiere a la preocupación por una estrecha relación entre reguladores y regulados, la cual puede ocasionar un fenómeno de captura del regulador, quien, en lugar de priorizar el interés general, protegerá los intereses de los regulados. Este enfoque también ha sido criticado por inhibir la productividad. Se ha postulado que esto es una consecuencia inevitable de las reglas inflexibles y complicadas que se generan para controlar la contaminación. Finalmente, este enfoque es de difícil aplicación en los países en vías de desarrollo, pues las instituciones reguladoras -junto con las instituciones judiciales, legislativas y de recopilación de datos- son, en general, mucho más débiles que en los países industrializados (Jacobson, 2002).

Por lo anterior, se tiende a favorecer más a las estrategias basadas en el mercado, como los incentivos económicos para reducir la contaminación atmosférica. Estas políticas ponen un precio explícito o implícito sobre las emisiones contaminantes. Los tres principales ejemplos son: 1) las tarifas sobre emisiones, en las que las empresas pagan una tarifa por los contaminantes que emiten a la atmósfera: a mayor cantidad de emisiones, mayores costos para las empresas; 2) los bonos comercializables, que son documentos que otorgan "permisos" para que las empresas emitan cierta cantidad de contaminantes (en caso de que no sobrepasen esa cantidad, pueden intercambiar sus remanentes con otras empresas y obtener un beneficio económico); y 3) los impuestos ambientales, que son simplemente impuestos sobre los 
insumos utilizados por los contaminadores o sobre los bienes y servicios producidos (Stavins, 2003).

Los incentivos económicos son más fáciles de aplicar que la regulación command and control porque operan a través de instituciones de recaudación de impuestos, en lugar de instituciones reguladoras y, muchas veces, las primeras son más efectivas que las segundas. Además, tienen dos tipos de impactos positivos: los fiscales, porque aumentan los ingresos del gobierno; y los ambientales, siempre y cuando sean efectivos en la reducción de la contaminación atmosférica. Pero estos dos impactos están inversamente relacionados y dependen de la elasticidad del bien gravado. Por ejemplo, si la demanda de gasolina es inelástica, ${ }^{1}$ un impuesto a este producto generará ingresos, pero no reducirá significativamente su consumo o las emisiones contaminantes. Por otro lado, si la demanda de gasolina es elástica, ${ }^{2}$ el impuesto generará relativamente pocos ingresos, pero reducirá su consumo y, presumiblemente, las emisiones vehiculares (Stavins, 2003).

Asimismo, los incentivos económicos, en especial los impuestos ambientales, implican una serie de desventajas. La más importante es que no crean estímulos para reducir las emisiones per se, sino sólo para limitar las compras de un bien relacionado con las emisiones. Otra desventaja es que pueden afectar las actividades no focalizadas. Por ejemplo, un impuesto sobre el carbón destinado a reducir las emisiones derivadas de la combustión afectará a los fabricantes de productos químicos que utilizan el carbón como materia prima, no como combustible. Finalmente, los impuestos ambientales pueden tener impactos distributivos adversos; es decir, pueden tener un efecto más severo en los hogares pobres que en los ricos (Stavins, 2003).

Por lo anterior, los incentivos económicos no han reemplazado del todo al enfoque command and control. Incluso cuando estos instrumentos de mercado se han utilizado en su forma más pura y con cierto éxito, como en el caso de los bonos comercializables en Estados Unidos, no siempre han funcionado como se esperaba (Stavins, 2003). Sin embargo, existe otra alternativa de solución al problema de la contaminación atmosférica que, además de combinar algunos elementos de los enfoques revisados, contiene un análisis exhaustivo de datos geográficos, demográficos, climáticos y de calidad del aire para generar medidas de control de la contaminación atmosférica: los programas urbanos de gestión de la calidad del aire.

Según Miranda et al. (2015), aunque esta alternativa de solución es determinada por cada ciudad con base en sus prioridades, los componentes

${ }^{1}$ Los aumentos del precio de la gasolina tienen poco efecto en su demanda.

${ }^{2}$ Los aumentos del precio de la gasolina reducen significativamente su demanda. 
de un programa urbano de gestión de la calidad del aire (PUGCA) deben considerar, al menos: a) la puesta en marcha de una red de monitoreo de calidad del aire; $b$ ) la elaboración de un inventario de emisiones; $c$ ) el modelado de la calidad del aire y la predicción de impactos; y d) la definición de medidas de control. El objetivo general de un PUGCA es garantizar que la calidad del aire cumpla con los requisitos especificados y contribuir al logro de los compromisos que el gobierno adoptó para reducir la contaminación atmosférica.

La información sobre el estado actual de la calidad del aire surge a partir de un monitoreo y modelado sistemático, pues la identificación de las fuentes de contaminación es clave en el diseño de un PUGCA. Después es necesario delinear las medidas de control, las cuales se deben prescribir a detalle. Por lo general, el PUGCA contiene documentos anexos con una descripción detallada de la implementación de cada medida propuesta, así como la lista de agencias responsables de dicha implementación, los plazos, el presupuesto planificado y un calendario que indica el porcentaje de cumplimiento de metas. Al respecto, el Esquema 1 ilustra los diferentes componentes que deben incluirse en un PUGCA.

Cabe señalar que el análisis de las siguientes dos etapas del proceso de políticas públicas, implementación y evaluación, se concentrarán exclusivamente en los programas urbanos de gestión de la calidad del aire; lo anterior por las siguientes razones: $a$ ) es la alternativa de solución que se adoptó en la ZMVM para reducir la contaminación atmosférica; b) dicha alternativa contempla algunos de los instrumentos del enfoque command and control; y c) los incentivos económicos son todavía una alternativa en proceso de consolidación, pues hasta el segundo semestre de 2018 se lanzará formalmente el mercado de carbono en nuestro país (El Economista, 2017).

\section{Implementación}

Entre la formulación de soluciones y la evaluación de sus resultados se encuentra la implementación. Se trata de una etapa en la que se aplican los instrumentos y los recursos que han sido previstos en el diseño de la política. La finalidad de todas las actividades que se desarrollan en esta etapa es alcanzar los objetivos previstos o, al menos, acercarse a ellos. La implementación es la etapa del proceso posterior a la aprobación de una ley, plan o programa diseñado para resolver el problema identificado. Vista de esa manera, significa la ejecución de una política pública, donde se conjuntan actores, procedimientos y técnicas para poner en marcha las acciones adoptadas (O'Toole, 2004). 


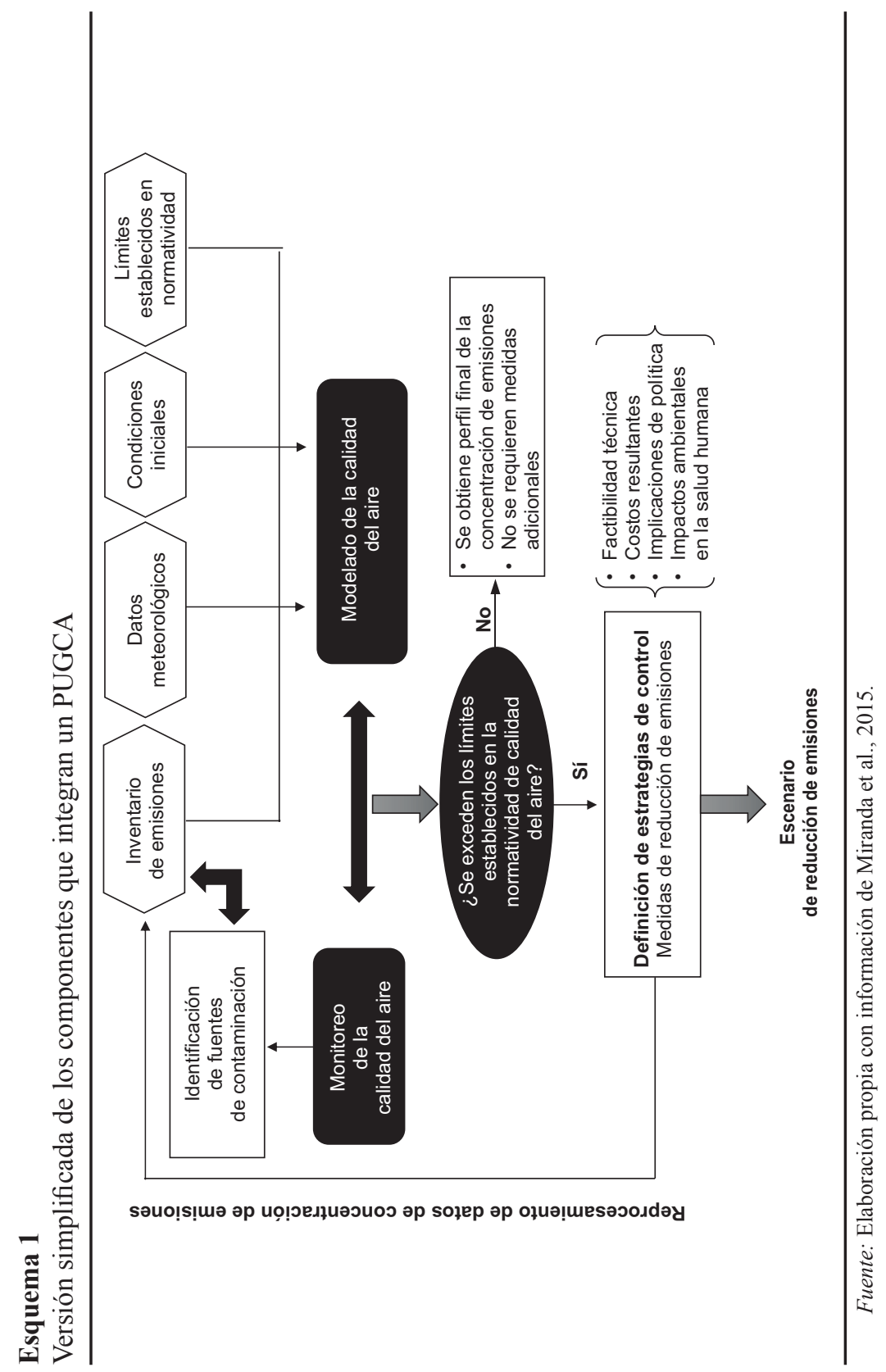


Anteriormente, la implementación era considerada una etapa de menor rango que las etapas de formulación y evaluación de políticas. Por ello, recibió menor atención tanto de los académicos como de los gerentes públicos. Se tenía la idea de que la implementación consistía básicamente en la "aplicación" de un diseño de política previamente elaborado. Desde esta perspectiva, los recurrentes problemas que se manifestaban en la puesta en marcha de una política respondían a dos tipos de situaciones: la implementación no se "ajustaba" a lo establecido en el diseño o -cuando la respuesta anterior no era suficiente- el diseño no había previsto situaciones, factores o procesos que serían objeto de rediseño (O’Toole, 2004).

Sin embargo, recientemente se ha postulado que la implementación es en sí misma una fuente de dificultades que no pueden solucionarse "ajustándose" mejor a lo planificado. Dichas investigaciones sugieren que la mayor parte de las fallas de implementación responden a la complejidad que tienen las rutinas, actividades e interacciones consideradas como ordinarias y por lo tanto poco importantes (O'Toole, 2004). En virtud de lo anterior y con el fin de resaltar algunos elementos del diseño e implementación de los PUGCA, a continuación se examinará cómo han sido instrumentados sus componentes en otros países y regiones.

\section{Componentes de los programas urbanos de gestión de la calidad del aire}

\section{Red de monitoreo de calidad del aire}

Uno de los componentes más importantes de un PUGCA es una red de monitoreo (Sivertsen, 2008). El monitoreo proporciona información sobre el estado actual de la calidad del aire. También ayuda a evaluar la efectividad de las políticas existentes. Por ejemplo, la red de monitoreo de calidad del aire en el área del Gran Londres ha sido diseñada para realizar análisis de correlación espacial (Ma, Richards, Ghanem, Guo y Hassard, 2008). Otros países de la Unión Europea administran su red de monitoreo a través del Centro Europeo sobre Contaminación del Aire y Cambio Climático (Holman, Harrison y Querol, 2015). Por su parte, en Estados Unidos la redes de monitoreo fueron diseñadas siguiendo pautas de la Oficina de Planificación y Normas de Calidad del Aire. Los datos de monitoreo se analizan para pronosticar el índice de calidad del aire en más de 300 ciudades (Lamsal et al., 2015).

En cuanto a los países en desarrollo, las redes de monitoreo de calidad del aire sirven para asegurar un cumplimiento efectivo de la normatividad. 
Por ejemplo, en Sudáfrica operan 94 estaciones de monitoreo de la calidad del aire en todo el país, lo que garantiza el cumplimiento normativo (Naiker, Diab, Zunckel y Hayes, 2012). En China se han establecido docenas de estaciones de monitoreo de la calidad del aire para evaluar, en tiempo real, las concentraciones de contaminantes atmosféricos en las megaciudades (Chan y Yao, 2008). En India, la red nacional de monitoreo de la calidad del aire tiene 342 estaciones de monitoreo en 127 ciudades. Además, las juntas estatales de control de la contaminación operan sus propias estaciones de monitoreo (Gupta, 2008).

\section{Elaboración de un inventario de emisiones}

Un inventario de emisiones puede definirse como una recopilación de datos de las fuentes de emisión y los contaminantes emitidos para una determinada área geográfica y escala temporal. Dicho inventario es importante para desarrollar una estrategia de control de emisiones, pues genera datos sobre las cantidades de contaminantes que se emiten y de dónde provienen. Los datos, identificados geográficamente y temporalmente, se utilizan como insumo de información para los modelos de gestión de la calidad del aire (Borge et al., 2014).

Los inventarios de emisiones nacieron en 1979 gracias al Convenio sobre Contaminación Transfronteriza a Larga Distancia, del cual derivaron el Programa de Cooperación para la Vigilancia y Evaluación de los Contaminantes Atmosféricos en Europa (EMEP) y el inventario Corinair. A partir de 1993, EMEP y Corinair se acoplaron dando como resultado la iniciativa AQMEII, Air Quality Model Evaluation International Initiative. Gracias a esta iniciativa se han analizado y evaluado estadísticamente las emisiones de Norteamérica y Europa desde el año 2006 (Kuenen, Visschedijk, Jazwicka y Denier van der Gon, 2014). La estimación de contaminantes atmosféricos también se volvió importante en Asia, donde se han producido dramáticas variaciones espaciales y temporales de emisiones en las últimas décadas. ${ }^{3}$ Estos inventarios también son comunes en África y América Latina (Borge et al., 2014).

${ }_{3}$ Por ello, se elaboró un inventario regional de emisiones. El periodo de observación fue de 1980 a 2020, considerando emisiones históricas y proyecciones futuras. Para todos los tipos de contaminantes, China fue el mayor contribuyente a las emisiones asiáticas, y sus tasas de crecimiento también fueron las mayores debido al continuo aumento en su consumo de energía. El segundo contribuyente fue India, cuyas emisiones también crecieron rápidamente entre 2000 y 2008. Las emisiones de Japón, Corea del Sur y Taiwán fueron menos significativas debido a una regulación más efectiva (Kurokawa et al., 2013). 


\section{El modelado de la calidad del aire y la predicción de impactos}

El modelado de la calidad del aire desempeña un papel importante en la formulación de estrategias de control de la contaminación atmosférica, pues proporciona directrices para el diseño de un PUGCA. Su principal objetivo es analizar el comportamiento de los contaminantes atmosféricos, los procesos meteorológicos, así como los cambios físicos y químicos que se producen en la atmósfera. En este aspecto, los modelos de calidad del aire son el nexo entre los inventarios de emisiones y las estrategias de control de la contaminación. A través de ellos es posible planear escenarios para la implementación de políticas de reducción de emisiones, así como verificar el cumplimiento de las mismas (Touma, Isakov, Ching y Seigneur, 2006). ${ }^{4}$

Según Miranda et al. (2015), los modelos eulerianos son los más utilizados en Europa; para su funcionamiento sólo requieren datos de las emisiones de diversos sectores de actividad, variables meteorológicas, así como condiciones iniciales y de frontera. De acuerdo con estos autores, más del $40 \%$ de los PUGCA europeos utiliza modelos eulerianos, seguido de modelos gaussianos (20\%). En Estados Unidos, la Agencia de Protección Ambiental recomienda el modelo de receptores Aermod (modelo de dispersión en estado estacionario), que se utiliza con fines regulatorios. Otro modelo recomendado por esta agencia es CALPUFF, que se utiliza para calcular el transporte de contaminantes de largo alcance (distancias de $50 \mathrm{~km}$ a varios cientos de kilómetros) (Cimorelli et al., 2005).

Algunos modelos de calidad del aire incluyen un enfoque de optimización que toma en cuenta aspectos como la predicción de impactos en la salud y el medio ambiente. Por ejemplo, en la zona metropolitana de Londres se utiliza el Urban Scale Integrated Assessment Model (USIAM), mientras que en Bélgica se emplea con más frecuencia el modelo AURORA (Air Quality Modeling in Urban Regions using an Optimal Resolution Approach (Miranda et al., 2015).

${ }^{4}$ De acuerdo con EEA (2011), para medir la calidad del aire existen: 1) modelos gaussianos, que asumen a la distribución de la concentración de contaminantes con una distribución gaussiana; 2) modelos eulerianos, donde un área se divide en celdas, tanto en la dirección horizontal como vertical; las concentraciones se calculan en posiciones geográficas fijas y en momentos temporales concretos, basándose en concentraciones iniciales, nuevas emisiones, transporte hacia dentro y hacia afuera de cada celda, así como dilución y reacciones químicas; y 3) modelos de receptores, los que comienzan con la observación de las concentraciones que llegan al receptor, para posteriormente definir las fuentes que las provocan. 


\section{La definición de medidas de control}

El último paso en el diseño de un PUGCA es el desarrollo de medidas de control de emisiones. Dichas medidas de reducción pueden ser clasificadas como técnicas y no técnicas. Las técnicas son las denominadas "end of pipe technologies", que no modifican las fuerzas motrices de las emisiones, ni modifican la composición estructural de los sistemas productivos. Son medidas para la eliminación de los contaminantes una vez producidos, justo antes de salir de la fuente o incluso después de salir. Se trata de innovaciones incrementales en las que se emplea una tecnología depuradora al final del proceso, por ejemplo, filtros en chimeneas.

Por su parte, las medidas no técnicas reducen las fuerzas motrices antropogénicas y pueden estar relacionadas con cambios en el comportamiento de las personas (educación y concientización medioambiental o compartir automóviles), así como en tecnologías que reducen la demanda de energía o disminuyen el consumo de combustibles fósiles (el uso de autos eléctricos) (Levy, Wilson y Zwack, 2007). Con el fin de examinar si en la ZMVM se han seguido las mismas pautas que las regiones y ciudades analizadas han usado para el diseño e implementación de sus PUGCA, veamos a continuación los componentes de Proaire.

\section{Programa para Mejorar la Calidad del Aire (Proaire)}

En la década de los sesenta los habitantes de la ZMVM comenzaron a tomar conciencia del problema de la contaminación atmosférica, pero no se contaba con el conocimiento técnico ni con la estructura institucional para controlarla. No obstante, en 1966 la Secretaría de Salubridad y Asistencia integró la primera red de monitoreo con equipos para medir dióxido de azufre $\left(\mathrm{SO}_{2}\right)$ y partículas suspendidas. Más tarde, en los ochenta, se amplió esta red de monitoreo para establecer el Programa de Contingencias Ambientales y el Programa de un Día sin Auto (CAM, 2013, p. 113).

Gracias a estas acciones, en 1990 fue posible instrumentar el Programa Integral Contra la Contaminación Atmosférica (PICCA). Este programa fue el primer esfuerzo sistemático para abatir los niveles de contaminación en la ZMVM. Posteriormente, en 1992 se creó la Comisión para la Prevención y Control de la Contaminación Ambiental en la Zona Metropolitana del Valle de México, la cual acordó la implementación del Programa para Mejorar la Calidad del Aire en el Valle de México 1995-2000 (Proaire). A diferencia del PICCA, el Proaire se planteó por primera vez objetivos cuantitativos en 
materia de calidad del aire, con un conjunto de estrategias e instrumentos orientados a la industria, los vehículos y la planeación del desarrollo urbano, por lo que podría considerarse la primera experiencia en la implementación de un PUGCA en la ZMVM. Enseguida se presentan los componentes de Proaire.

\section{Red de monitoreo de la calidad del aire}

La medición de la calidad del aire en la ZMVM se inició en 1966, para lo cual se integró la primera red de monitoreo con cuatro estaciones de equipos manuales para medir dióxido de azufre, partículas suspendidas, polvo y acidez. Las estaciones se localizaron en el centro de la ciudad, Tlalnepantla, el aeropuerto y Tacuba. Más tarde, en 1973 se adquirieron 22 equipos automáticos de monitoreo que, sumados a los cuatro anteriores, conformaron una red de 26 estaciones. En los noventa se instalaron otras seis estaciones en Cuajimalpa, Tlalpan, Tláhuac, Chapingo, Villa de las Flores y Atizapán, con lo cual la cobertura creció de 26 a 32 estaciones. Asimismo, para extender la capacidad de medición de la red, se instalaron analizadores de ozono y óxidos de nitrógeno para tener un sistema de medición más moderno de interacción y formación de contaminantes secundarios (Zuk, Tzintzun y Rojas, 2007).

Al finalizar la modernización de este sistema, y en el marco de diversos procesos de descentralización, la federación transfirió la responsabilidad de la red de monitoreo de calidad del aire a las autoridades del Distrito Federal (Zuk, Tzintzun y Rojas, 2007). En la actualidad, esta red tiene 49 estaciones con 175 aparatos automáticos y 39 manuales instalados. Su gestión está a cargo de la Secretaría de Medio Ambiente del Gobierno de la Ciudad de México, que, a través de su Dirección de Monitoreo Atmosférico, se encarga de operar y administrar el Sistema de Monitoreo Atmosférico de la ZMVM (Simat). ${ }^{5}$

El Simat cuenta con un programa preventivo para garantizar la correcta operación de los equipos y la confiabilidad de sus registros, así como para evaluar la efectividad de las actividades realizadas; esto provee las bases

5 El Simat contiene los siguientes subsistemas: la Red Automática de Monitoreo Atmosférico (RAMA) y la Red Manual de Monitoreo Atmosférico (Redma), que determinan la concentración de los contaminantes criterio. También la Red de Meteorología y Radiación Solar (Redmet), que mide los parámetros meteorológicos para evaluar la calidad del aire. Además, opera la Red de Depósito Atmosférico (Redda), que colecta muestras de lluvia para analizar su contenido. El Simat también cuenta con un Centro de Información de la Calidad del Aire (CICA), donde se concentran los datos procedentes de dichas redes. 
para establecer acciones correctivas si se descubren deficiencias. Lo anterior permite que se generen reportes sobre el estado actual de la contaminación atmosférica, los cuales se presentan en datos y gráficas en el sitio web de la Dirección de Monitoreo Atmosférico. Por todo lo anterior, algunos consideran que el Simat cumple de manera satisfactoria su tarea de monitorear la calidad del aire en la ZMVM (Perevochtchikova, 2009).

\section{Elaboración de un inventario de emisiones}

En el inventario de emisiones de la ZMVM se estiman 90 categorías de emisión, tanto de contaminantes criterio ${ }^{6}$ como de compuestos tóxicos. Este inventario incluye como categorías de estimación a comercios y servicios regulados, cambios de uso de suelo, tratamiento de composta y tiraderos a cielo abierto. Gracias a este inventario también se obtienen datos de emisiones de gases de efecto invernadero. En ese sentido, el inventario pretende dar un panorama de los factores que afectan la calidad del aire y la cuantificación de los contaminantes que se liberan a la atmósfera por tipo de fuente (SMA, 2016a, pp. 12-13).

Así, en cuanto a las emisiones de precursores de ozono,

En el año 2014 se registraron 138454 toneladas anuales de NOX y 360123 de COV. Respecto a las partículas, el inventario de emisiones de la ZMVM estimó en 31431 toneladas anuales la emisión de $\mathrm{PM}_{10}$, de las cuales $41 \%$ corresponden a $\mathrm{PM}_{2.5}$. En lo que se refiere a los gases de efecto invernadero, se emitieron alrededor de 56.2 millones de toneladas de $\mathrm{CO}_{2}$ equivalente y 1793 toneladas de carbono negro [SMA, 2016a, p. 14].

De acuerdo con la Secretaría de Medio Ambiente (SMA),

[...] en cada actualización de los inventarios se incorporan mejores prácticas para aumentar el control de calidad de los cálculos y resultados, así como la revisión y evaluación de actualizaciones metodológicas para la recopilación de información que ayuda a agregar nuevos sectores de importancia [SMA, 2016a, p. 16].

En el Cuadro 1 se ofrece una descripción general de los contaminantes criterio y por tipo de fuente, los cuales contribuyen con un alto porcentaje de la contaminación del aire en la ZMVM.

${ }^{6}$ La OMS (2014) ha clasificado a las siguientes sustancias como contaminantes criterio: dióxido de azufre $\left(\mathrm{SO}_{2}\right)$, ozono $\left(\mathrm{O}_{3}\right)$, óxidos de nitrógeno (NOx), monóxido de carbono $(\mathrm{CO})$, partículas finas (PM 2.5 y PM 10) y los compuestos orgánicos volátiles (COV). 


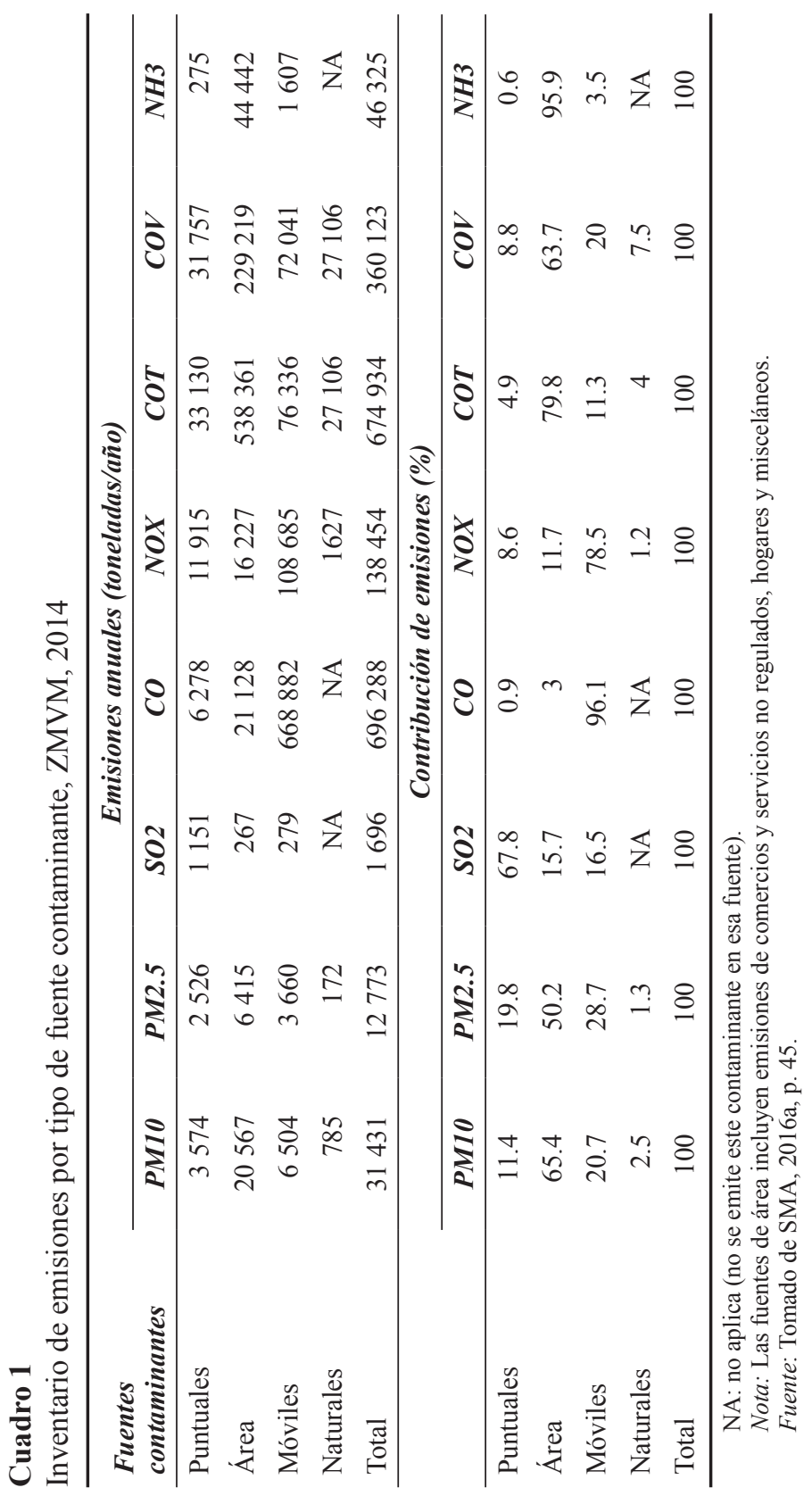




\section{El modelado de la calidad del aire y la predicción de impactos}

Para el análisis de la contaminación atmosférica en la ZMVM, la SMA ha desarrollado modelos eulerianos de celdas de $1 \mathrm{~km}^{2}$ de tamaño. ${ }^{7}$ Gracias al inventario de emisiones, todas las fuentes contaminantes son distribuidas temporal y espacialmente en dichas celdas. Así, la modelación de la calidad del aire relaciona las emisiones de la ZMVM con las condiciones meteorológicas y estima las concentraciones de ozono, de partículas o de otros contaminantes. Esta modelación también requiere datos geográficos y meteorológicos que se obtienen del Simat.

De esta forma, con el inventario de emisiones distribuido en celdas y con la modelación matemática se efectúa la caracterización de emisiones de los contaminantes criterio. De acuerdo con la SMA (2016b), desde 2014 se observa una disminución en los contaminantes primarios como dióxido de azufre, óxidos de nitrógeno y $\mathrm{PM}_{10}$. No obstante, en los contaminantes secundarios como el ozono y las $\mathrm{PM}_{2.5}$, la disminución ha sido menor. Además, no se han observado cambios importantes en los promedios anuales de los contaminantes, lo que podría indicar una condición de equilibrio entre las acciones de control y el volumen de emisiones. Para la SMA (2016b), el equilibrio anterior puede estar relacionado con un crecimiento continuo de la ZMVM, la cual mantiene una demanda creciente de energía, así como de transporte y servicios, y ello se traduce en un incremento en la generación de contaminantes. A continuación, se presentan las medidas de control de la contaminación atmosférica contempladas en el Proaire.

\section{La definición de medidas de control}

El Programa para Mejorar la Calidad del Aire (Proaire) 2011-2020 está constituido por ocho estrategias, las cuales contienen un total de 81 medidas de gestión de la calidad del aire (CAM, 2013). Dichas estrategias son las siguientes:

1) Ampliación y refuerzo de la protección de la salud

2) Disminución estructural del consumo energético de la ZMVM

7 Además, la SMA cuenta con los siguientes modelos matemáticos: MM5, WRF y CALMET, para estimar las condiciones meteorológicas; MCCM, WRF-Chem y WRF-CMAQ, para el análisis de la dinámica de las concentraciones de contaminantes a escala regional; y CALPUFF y AERMOD, para la simulación de la dispersión de contaminantes en escala urbana y microescala. 
3) Calidad y eficiencia energéticas en todas las fuentes

4) Movilidad y regulación del consumo energético del parque vehicular

5) Cambio tecnológico y control de emisiones

6) Educación ambiental, cultura de la sustentabilidad y participación ciudadana

7) Manejo de áreas verdes y reforestación urbana

8) Fortalecimiento institucional e investigación científica

Ahora bien, la gama de medidas específicas para la gestión de la calidad del aire es muy amplia (en total son 81); por esta razón, y con el propósito de analizar algunas de ellas, en el Cuadro 2 se presentan aquellas acciones que la Comisión Ambiental Metropolitana (CAM, 2013, pp. 187-188) ha seleccionado para construir los escenarios de reducción de emisiones de la ZMVM.

Del análisis del Cuadro 2 se desprende que tres estrategias de Proaire (6. Educación ambiental, cultura de la sustentabilidad y participación ciudadana; 7. Manejo de áreas verdes y reforestación urbana; y 8 . Fortalecimiento institucional e investigación científica) no han sido tomadas en cuenta para construir los escenarios de reducción de emisiones de la ZMVM. La exclusión de algunas de ellas puede estar justificada, ya que las medidas que contemplan dichas estrategias no inciden de forma directa en la emisión de contaminantes. Además, su efecto en las mejoras de la gestión de la calidad del aire es de largo plazo. Se trata de acciones que buscan fomentar la educación ambiental y la participación ciudadana en la elaboración de los planes y programas que regulan el funcionamiento de la ZMVM. En el mismo sentido se encuentran aquellas acciones dirigidas al desarrollo de estudios e investigación científica y tecnológica que permitan comprender mejor el funcionamiento del ecosistema metropolitano.

No obstante, llama la atención que no hayan sido incluidas las medidas contempladas en la estrategia 7: "Manejo de áreas verdes y reforestación urbana", pues la ZMVM está aún lejos de la proporción recomendada internacionalmente de áreas verdes, lo cual contribuye al empobrecimiento de la calidad del entorno urbano. Sin duda, un mayor crecimiento de la cobertura vegetal en la ZMVM coadyuvaría, en el corto plazo, a atenuar los efectos de la contaminación atmosférica. En ese sentido, estas y otras carencias contempladas en el diseño e implementación de Proaire representan áreas de oportunidad que vale la pena señalar y, por supuesto, atender. De ello se ocupará la siguiente sección. 


\section{Retos en el diseño e implementación de Proaire}

\section{Red de monitoreo de calidad del aire}

Como se señaló en la sección anterior, la operación de la red de monitoreo del Simat se apega a los lineamientos que establecen las Normas Oficiales Mexicanas. Además, este sistema cuenta con un programa preventivo y correctivo para garantizar la correcta operación de estos equipos y la confiabilidad de sus registros. Todo ello garantiza la efectividad del monitoreo.

Pese a lo anterior, Perevochtchikova $(2009$, p. 530) considera que la red de monitoreo de calidad del aire de la ZMVM presenta varias áreas de oportunidad que es necesario atender para mejorar su funcionamiento, entre ellas las siguientes:

- Una heterogénea distribución espacial de las estaciones de monitoreo, que presenta mayor número de estaciones al norte de la ZMVM, por lo que no abarca de manera homogénea todas las áreas que conforman esta región y, por lo tanto, se corre el riesgo de no brindar información exacta para prevenir la exposición de la población a graves concentraciones de contaminantes.

- La red de monitoreo no incluye en su lista a ciertos compuestos contaminantes, tanto industriales como vehiculares.

- Finalmente, falta una mayor inversión en la modernización y el mantenimiento del equipo de muestreo; es necesario aumentar la cantidad de unidades móviles para atender casos de emergencia y para proyectos específicos de investigación científica.

\section{Elaboración del inventario de emisiones y modelado de la calidad del aire}

En una situación ideal, el diagnóstico de emisiones asentado en los diferentes inventarios se debe compilar en un solo conjunto de datos básicos y proporcionar la información necesaria para tomar medidas de control de la contaminación. Asimismo, dicho conjunto de datos se debe actualizar a medida que se recibe nueva información y debe contemplar las posibles limitaciones en el monitoreo de contaminantes. Lo anterior haría posible que las actualizaciones de los inventarios de emisiones estén disponibles a los pocos días, si no es que horas, de que se recibió nueva información de la red de monitoreo. En tal situación, los diferentes inventarios de emisiones serían 


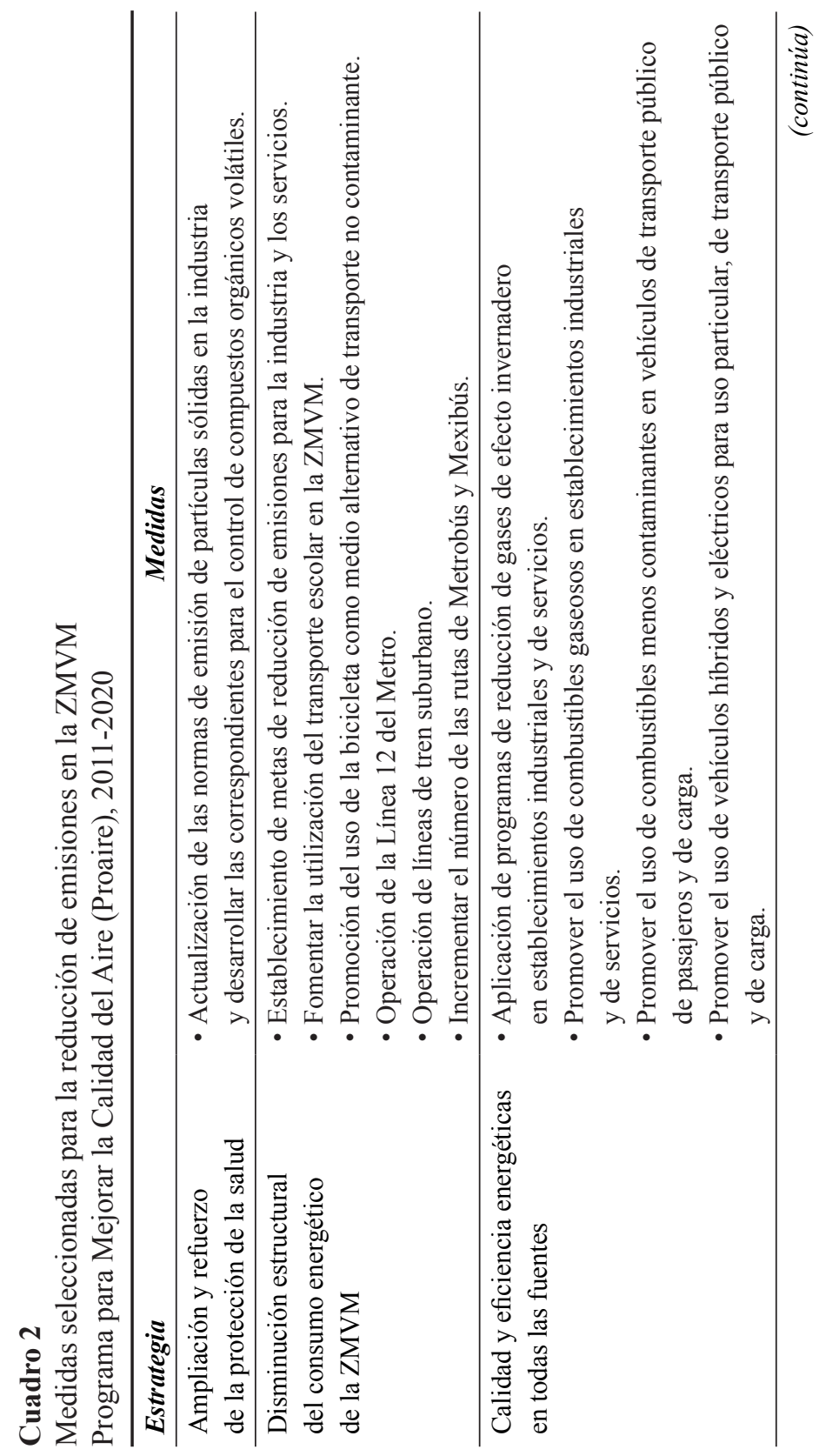




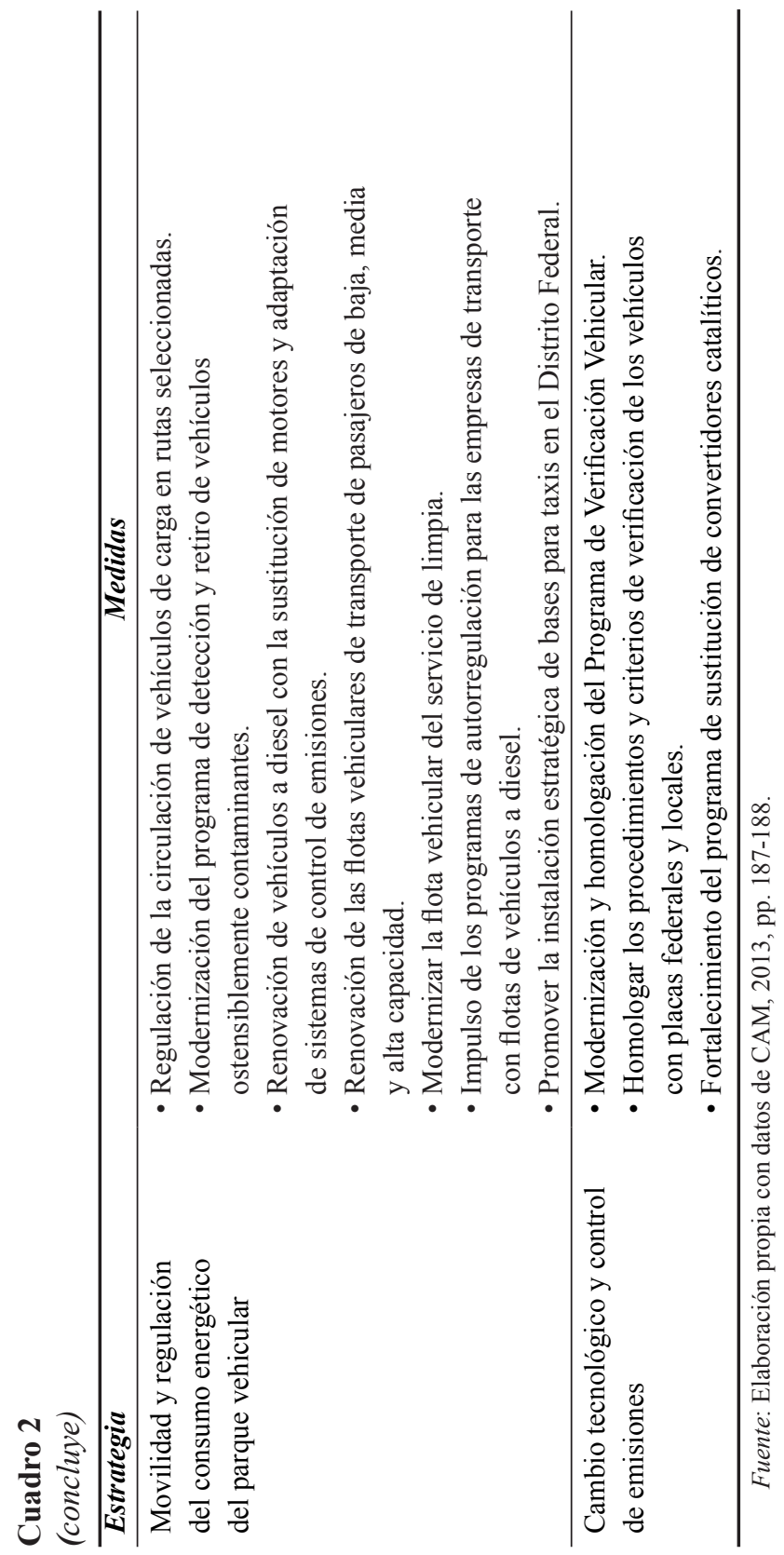


compatibles y comparables entre sí, brindando información casi en tiempo real, lo que permitiría disponer de información reciente sobre las emisiones y certidumbre en cuanto a su variabilidad.

Sin embargo, de acuerdo con Perevochtchikova (2009, p. 530), en la ZMVM no ha sido posible superar la incompatibilidad de los inventarios de emisiones. Además, la mayoría de sus estimaciones se basan en un pequeño número de mediciones que no representan adecuadamente toda la gama de procesos y prácticas operacionales. Esto limita la precisión y aumenta significativamente la incertidumbre de la estimación. Otro problema identificado es un desinterés por la optimización de las mediciones a escala local, lo cual provoca que se usen estimaciones de factores de asignación espacial para modelar la calidad del aire, en cuyo caso se provoca también incertidumbre en las estimaciones de contaminantes. Del mismo modo, el inventario de emisiones de la ZMVM se basa en valores promedio anuales, los cuales pueden estar muy lejos de los valores reales de los lapsos de una hora o día, que puedan ser de interés particular para monitorear la calidad del aire.

\section{La definición de medidas de control}

De acuerdo con la SMA (2016b), las medidas de control propuestas por Proaire lograron que en 2015 se mantuviera el cumplimiento de las Normas Oficiales Mexicanas para $\mathrm{SO}_{2}, \mathrm{CO}$ y NOX. Sin embargo, las concentraciones de $\mathrm{O}_{3}, \mathrm{PM}_{10}$ y $\mathrm{PM}_{2.5}$ continuaron superando los valores de dichas normas, pese a las medidas establecidas. Por otro lado, en marzo de 2016, debido a la situación extrema de las condiciones atmosféricas y de la concentración de contaminantes, la Comisión Ambiental de la Megalópolis (CAM) activó la Fase I de contingencia ambiental atmosférica por $\mathrm{O}_{3}$ en la ZMVM. Al respecto, la CAM informó que se alcanzaron 203 puntos IMECA, y por ello decretó la primera contingencia ambiental desde 2002. Dicha decisión implicó que dejara de circular casi 40\% del parque vehicular de la ZMVM. Además, se solicitó a la población limitar actividades deportivas, cívicas, sociales, de recreo u otras al aire libre.

En virtud de lo expuesto, es posible advertir que las medidas contempladas en el Programa para Mejorar la Calidad del Aire (Proaire) 2011-2020 no han resultado del todo eficaces. Sin embargo, para sustentar esta afirmación es conveniente recurrir a la última etapa del proceso de políticas públicas: la evaluación. 


\section{Evaluación}

Los efectos de una política pública pueden ser los esperados, pero también pueden ser mucho menos de los que se pretendía lograr o, incluso, producir un efecto no deseado. Si alcanza sus objetivos, se dirá que fue exitosa en su diseño e implementación. Lo contrario llevará a identificarla como una solución fallida. Así, para confirmar el éxito o fracaso de una política pública se requiere evaluarla. En ese sentido, la evaluación se entiende como la etapa del proceso en la cual se llevan a cabo actividades de observación, medición, análisis e interpretación de resultados de una intervención pública, con el objeto de alcanzar un juicio valorativo sobre su utilidad social (Dunn, 2004).

Asimismo, la evaluación se lleva a cabo con dos propósitos principales: para aprender de la experiencia, extrayendo lecciones que puedan aplicarse con el fin de mejorar el diseño y la implementación de las políticas públicas; y para la rendición de cuentas al público y/o a quienes han aportado los recursos utilizados. De esta forma, la evaluación permite identificar lo que ha funcionado bien y lo que ha fallado. Si las políticas públicas se implementaran siempre de acuerdo con las intenciones, y si no hubiera efectos no buscados, la evaluación no sería útil, excepto para confirmar el logro de los objetivos. En ese tipo de escenario no habría incertidumbre. Pero en el mundo real las políticas no siempre pueden implementarse conforme a las intenciones iniciales (Dunn, 2004).

Además, los problemas ambientales (como la contaminación atmosférica) tienen algunas características que los hacen difíciles de resolver. Por ejemplo, tienden a ser muy complejos. Un cambio en un aspecto físico o químico de un ecosistema generalmente afecta a otras partes de dicho ecosistema, y la complejidad se incrementa aún más por las interacciones humanas, sociales, políticas y económicas. Por otro lado, los problemas ambientales a menudo conectan regiones geográficamente remotas. Las emisiones de dióxido de azufre de una planta de electricidad en una ciudad pueden generar afectaciones en la salud a pobladores de otra urbe. Finalmente, la formulación de soluciones a los problemas ambientales requiere de mucho tiempo y conocimiento científico, pero ambos recursos son siempre limitados (Mickwitz, 2003).

Hay diferentes tipos de evaluaciones que pueden utilizarse. Entre ellas está la evaluación de resultados, que se centra en las modificaciones logradas en un conjunto de variables-objetivo (por ejemplo, las concentraciones de contaminantes). En ese sentido, la forma adecuada de evaluar el logro de resultados del Proaire sería analizar, a lo largo del tiempo, el nivel de 
concentraciones de los principales contaminantes emitidos a la atmósfera de la ZMVM y contrastar dichos niveles con los límites establecidos por las normas de calidad del aire (Norma Oficial Mexicana, NOM). Sin embargo, por razones de espacio es complicado presentar un análisis exhaustivo de cada uno de ellos, por lo que se tratará exclusivamente el caso del ozono.

Usualmente, el ozono es uno de los contaminantes criterio más utilizados para elaborar índices de calidad del aire que comunican a la población el estado de la calidad del aire y los riesgos asociados. Dicho índice es un instrumento de difusión empleado para evitar el uso de unidades de medición confusas para la población. A partir del siglo pasado, "el índice oficial de contaminación del aire en México ha sido el Índice Metropolitano de Calidad del Aire (IMECA)" (Correa García, 2011, p. 74). Desde que se inició el monitoreo sistemático del $\mathrm{O}_{3}$ en la ZMVM, todos los años se han rebasado los límites definidos por la NOM. Durante los años noventa se alcanzaron máximos históricos con concentraciones de casi tres veces el valor de la norma de 110 partes por millón (ppm). Pero en 2014 el valor límite del ozono disminuyó a 95 ppm; pese a ello, en 2016 se registró una concentración promedio horario de $150 \mathrm{ppm}$ en la atmósfera de la $\mathrm{ZMVM}^{8}$ (Gráfica 1).

Esta situación de mala calidad del aire ha sido una constante en la ZMVM. Al respecto, en marzo de 2016 fue activada una contingencia ambiental al registrarse una concentración de 203 puntos de ozono en el IMECA. Así, en cuanto al número de días en que se rebasan las normas de calidad del aire por ozono, es evidente que el problema de contaminación atmosférica en la ZMVM continúa siendo grave: no han disminuido del 60\% de 2015 a la fecha. ${ }^{9}$

A partir de este ejercicio de evaluación surge una pregunta interesante: ¿por qué razón Proaire no ha sido exitoso en el control de la contaminación atmosférica en la ZMVM? Es difícil delinear una respuesta a esta interrogante, pero a lo mejor se pueden identificar ciertas pistas en el diseño o la

8 Actualmente, el IMECA emplea una escala normalizada de 0 a 500 puntos, donde el valor 0 corresponde a la ausencia de contaminantes y el valor 100 a los valores límite establecidos en las NOM. Entre mayor es el valor del índice, mayor es la concentración del contaminante y por lo tanto el riesgo para la salud. Cuando el índice supera los 100 puntos, existe riesgo para la salud de los grupos más sensibles de la población; cuando rebasa los 150 puntos el riesgo se generaliza para toda la población. En el caso del ozono, el índice se calcula utilizando el valor máximo de cada hora (GDF, 2014, p. 51).

9 Por ejemplo, en 2015 se registraron 201 días con una calidad del aire mala para ozono ( $>100$ puntos); de éstos, en ocho ocasiones la concentración de contaminante alcanzó una condición muy mala (>150 puntos). Las cifras para 2016 fueron de 212 y 18, respectivamente; mientras que, en el mes de junio de 2017, se registraron 129 días con una calidad del aire mala (>100 puntos) y 10 con una calidad muy mala (>150 puntos) (GDF, 2017).

Estudios Demográficos y Urbanos, vol. 34, núm. 3 (102), 2019, pp. 631-663

doi: http://dx.doi.org/10.24201/edu.v34i3.1806 


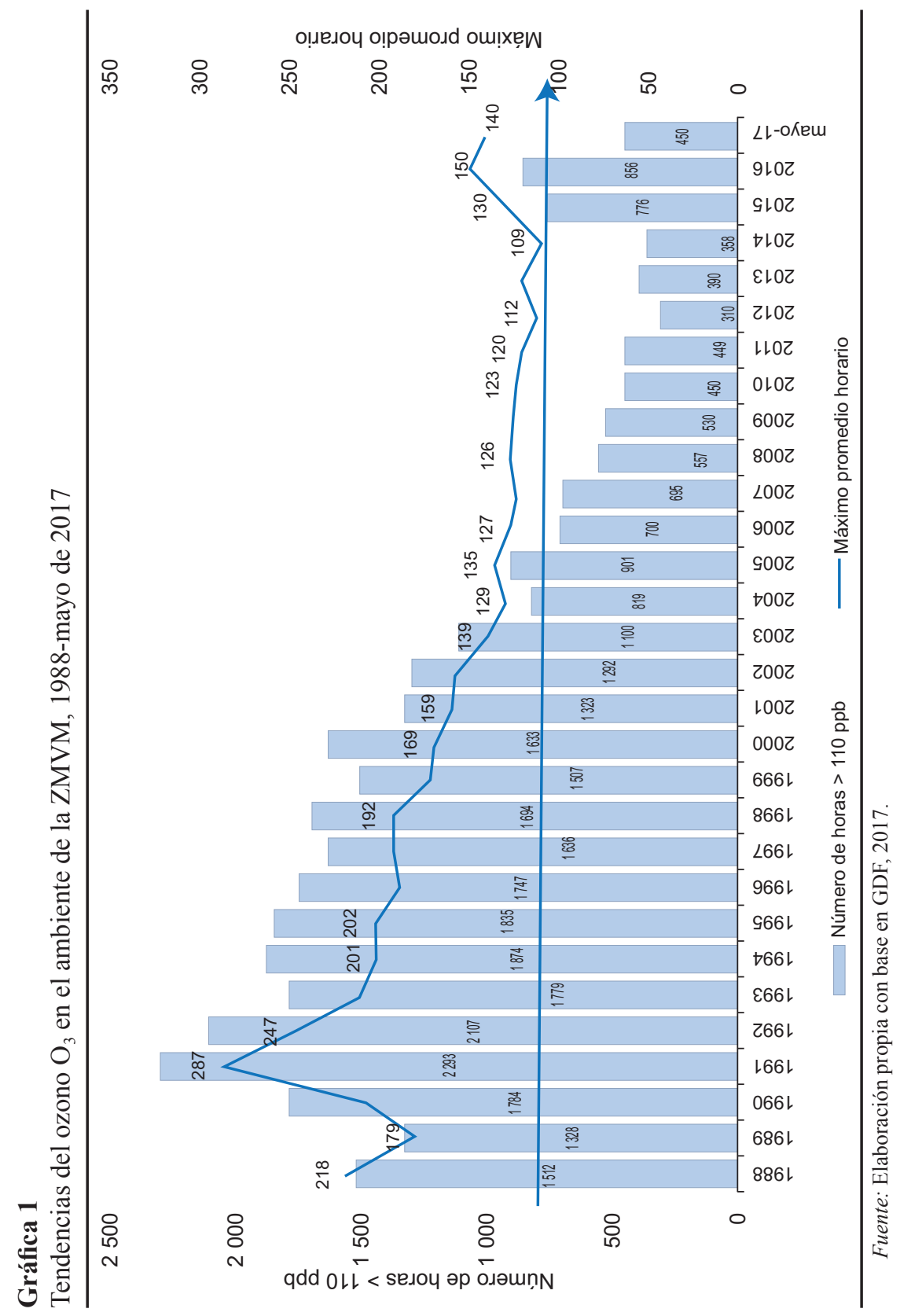


implementación de esta política pública. Al respecto, cabe señalar que las acciones instrumentadas en Proaire son similares a muchas de las estrategias de control de la contaminación atmosférica adoptadas en otras ciudades. En ese sentido, el Cuadro 3 presenta las similitudes y diferencias entre las medidas de control de la contaminación implementadas por Proaire y las que se han puesto en marcha en otras ciudades.

Algunas de las medidas incluidas en el Cuadro 3 se relacionan con adelantos tecnológicos para reducir las emisiones contaminantes o con restricciones al tráfico vehicular. Por otro lado, las medidas relacionadas con el mejoramiento o con nuevas formas de transporte dependen en gran medida de la aceptación pública y de la intención de la ciudadanía de renovar una flota vehicular vieja por opciones más ecológicas. En ese sentido, se puede observar que ciudades como Londres y Nueva York han logrado implementar completamente ambos tipos de medidas, pues han instrumentado con éxito todas las acciones referidas en el Cuadro 3.

En el caso de la ZMVM, con el establecimiento de Proaire sólo algunas medidas se han implementado completamente; por ejemplo, el mejoramiento de la calidad de los combustibles, la instalación de convertidores catalíticos, los programas de verificación vehicular, así como las normas de calidad del aire. Por otro lado, en la ZMVM no se han logrado poner en marcha medidas importantes como el establecimiento de zonas de cero emisiones y las zonas con restricciones para flujo o estacionamiento de vehículos (salvo algunos corredores peatonales en el Centro Histórico de la Ciudad de México, este tipo de medidas son inexistentes en la ZMVM). Por supuesto, tampoco existen sistemas de tarifas de congestión vehicular, medida que ha sido muy exitosa en Londres para la reducción de la contaminación atmosférica (Ma Richards, Ghanem, Guo y Hassard, 2008).

En la ZMVM también destaca que no se haya implementado de forma completa el uso de la bicicleta como transporte alternativo (dicha medida sólo existe en algunas colonias de tres delegaciones de la Ciudad de México), y tampoco las líneas de autobuses con carriles confinados (ha habido muchos problemas con la construcción de dichas líneas; actualmente existen seis, y por conflictos con los vecinos apenas comenzó a funcionar la Línea 7). Por su parte, la prohibición de circulación de vehículos pesados no es tan estricta, ya que los vehículos de más de 3.5 toneladas no circulan sólo entre las 7 y las 9 de la mañana, de lunes a viernes. En cuanto a la reducción de impuestos a la adquisición de vehículos ecológicos, en la ZMVM sólo se ha logrado la exención de aranceles a la importación y del impuesto al consumo, pero no así a las cuotas por el uso de autopistas urbanas, pago de tenencia o trámite de placas. 





En síntesis, en cuanto al establecimiento de medidas de control de la contaminación atmosférica, las acciones impulsadas por Proaire son poco parecidas a ciudades de vanguardia en esta materia, como Tokio, Londres y Nueva York, y comparten mayores similitudes con ciudades, de países emergentes como: Beijing y Hong Kong en China; Kanpur en India; y Ciudad del Cabo en Sudáfrica. En estas últimas ciudades varias medidas referidas en el Cuadro 3 están parcialmente implementadas o aún no se instrumentan.

\section{Conclusiones}

El objetivo de este artículo fue analizar los mecanismos de control de la contaminación atmosférica en la ZMVM. Al respecto, se utilizó el ciclo de políticas públicas para examinar el Programa para Mejorar la Calidad del Aire (Proaire), principal estrategia implementada en la ZMVM para atender este problema público. En ese sentido, la Secretaría del Medio Ambiente del Gobierno de la Ciudad de México, mediante una red de monitoreo, realiza mediciones de la calidad del aire de la ZMVM y, a través de diversas metodologías, elabora y actualiza un inventario de emisiones con la finalidad de conocer el origen, la cantidad y el tipo de emisiones que se generan y liberan a la atmósfera.

Así, este inventario se ha convertido en una de las herramientas más importantes de Proaire, a partir del cual se han instrumentado diversas medidas de control de la contaminación atmosférica. No obstante, se observó que dichas medidas no han logrado cumplir todos sus objetivos, ya que los niveles de ozono aún rebasan con frecuencia el valor de la norma mexicana vigente. Lo anterior podría tener relación con el hecho de que Proaire, en su diseño e implementación, no ha incluido algunas de las estrategias usualmente contempladas en los programas urbanos de gestión de la calidad del aire (PUGCA) de otras ciudades.

En ese sentido, se puede observar que ciudades que van a la vanguardia en materia de control de la contaminación, como Tokio, Londres y Nueva York, han logrado implementar con éxito casi todas las estrategias que convencionalmente se incluyen en un PUGCA, destacando algunas muy eficaces, como el mejoramiento o construcción de nuevas líneas de transporte público, así como la renovación de la flota vehicular por opciones más ecológicas, por ejemplo, autos eléctricos. En el caso de la ZMVM, sólo algunas medidas se han instrumentado completamente: el mejoramiento de la calidad de los combustibles, la instalación de convertidores catalíticos, los programas de verificación vehicular, así como la actualización de las normas de calidad del aire. 
Sin embargo, en la ZMVM no se han logrado poner en marcha medidas importantes como el establecimiento de zonas de cero emisiones, restricciones para el flujo de vehículos, ni sistemas de tarifas de congestión vehicular; tales medidas han sido muy exitosas en otras ciudades para controlar la contaminación atmosférica. Por otro lado, los incentivos económicos, como las tarifas sobre emisiones, los bonos comercializables y los impuestos ambientales, son todavía una alternativa en proceso de consolidación. En este contexto, el reto actual de la gestión de la calidad del aire en la ZMVM consiste en incorporar algunas de esas acciones o en diseñar una nueva generación de políticas públicas; de no hacerlo, existe el riesgo de que en el futuro inmediato empeore el problema de la contaminación atmosférica.

\section{Bibliografía}

Borge, R., Lumbreras, J., Pérez, J., De la Paz, D., Vedrenne, M., De Andrés, J. M. y Rodríguez, M. E. (2014). Emission inventories and modeling requirements for the development of air quality plans. Application to Madrid (Spain). Science of the Total Environment, 466-467, 809-819. Recuperado de https://www.sciencedirect.com/science/article/pii/ S0048969713008759

Campbell, H. (2010). A comparative framework for analyzing urban environmental policy. Journal of Comparative Policy Analysis: Research and Practice, 12(4), 373-394. Recuperado de https://www.tandfonline. com/doi/full/10.1080/13876988.2010.495506?scroll=top\&needAccess $=$ true

Comisión Ambiental Metropolitana (CAM). (2013). Programa para Mejorar la Calidad del Aire de la Zona Metropolitana del Valle de México 20112020. Ciudad de México: Comisión Ambiental Metropolitana.

Chan, C. K. y Yao, X. (2008). Air pollution in mega cities in China. Atmospheric Environment, 42(1), 1-42. Recuperado de https://www.science direct.com/science/article/pii/S1352231007007911

Cimorelli, A., Perry, S., Venkatram, A., Weil, J., Paine, R., Wilson, R., .... y Brode, R. (2005). Aermod: A dispersion model for industrial source applications. Part I: General model formulation and boundary layer characterization. Journal of Applied Meteorology, 44(5), 682-693. Recuperado de https://journals.ametsoc.org/doi/pdf/10.1175/JAM2227.1

Correa García, A. (2011). Los índices de calidad del aire: alcances y limitaciones. Conciencia Tecnológica, 42, 74-76. Recuperado de http://www. redalyc.org/pdf/944/94421442014.pdf 
Dunn, W. (2004). Public policy analysis. An introduction. Nueva Jersey: Pearson Prentice Hall.

Dutton, J. (1986). Understanding strategic agenda building and its implications for managing change. Scandinavian Journal of Management Studies, 3(1), 3-24. Recuperado de https://www.sciencedirect.com/ science/article/pii/0281752786900083

El Economista. (2017). Se formalizará mercado de bonos de carbono. El Economista. Ciudad de México, 5 de junio.

European Environment Agency (EEA). (2011). The application of models under the European Union's air quality directive: A technical reference guide. Copenhague, Dinamarca: EEA.

Gobierno del Distrito Federal (GDF). (2014). Calidad del aire en la Ciudad de México. Informe 2014. Ciudad de México: Secretaría del Medio Ambiente.

Gobierno del Distrito Federal (GDF). (2017). Sistema de monitoreo atmosférico. Ciudad de México. Disponible en http://www.aire.df.gob.mx/ default.php

Gupta, U. (2008). Valuation of urban air pollution: A case study of Kanpur city in India. Environmental and Resource Economics, 41(3), 315-326. Recuperado de https://link.springer.com/content/pdf/10.1007\%2 Fs10640-008-9193-0.pdf

Holman, C., Harrison, R. y Querol, X. (2015). Review of the efficacy of low emission zones to improve urban air quality in European cities. Atmospheric Environment, 111, 161-169. Recuperado de https://www.science direct.com/science/article/pii/S1352231015300145

Jacobson, M. (2002). Atmospheric pollution: History, science, and regulation. Cambridge: Cambridge University Press.

Jann, W. y Wegrich, K. (2007). Theories of the policy cycle. En F. Fischer y G. Miller (eds.), Handbook of public policy analysis: Theory, politics, and method (pp. 43-62). Florida: CRC Press.

Kelly, F. y Fussell, J. (2015). Air pollution and public health: Emerging hazards and improved understanding of risk. Environmental Geochemistry and Health, 37(4), 631-649. Recuperado de https://link.springer. com/article/10.1007/s10653-015-9720-1

Kuenen, J., Visschedijk, A., Jozwicka, M. y Denier van der Gon, A. (2014). TNO-MACC_II emission inventory; a multi-year (2003-2009) consistent high-resolution European emission inventory for air quality modelling. Atmospheric Chemistry and Physics, 14(20), 10963-10976. Recuperado de https://www.atmos-chem-phys.net/14/10963/2014/acp-14-10963-2014.pdf Kurokawa, J., Ohara, T., Morikawa, T., Hanayama, S., Janssens-Maenhout, 
G., Fukui, T., Kawashima, K. y Akimoto, H. (2013). Emissions of air pollutants and greenhouse gases over Asian regions during 2000-2008: Regional emission inventory in Asia (REAS), version 2. Atmospheric Chemistry and Physics, 13(21), 11019-11058. Recuperado de https:// www.atmos-chem-phys.net/13/11019/2013/acp-13-11019-2013.html

Lamsal, L. N., Duncan, B., Yoshida, Y., Krotkov, N., Pickering, K., Streets, D. y Lu, Z. (2015). US NO² trends (2005-2013): EPAAir Quality System (AQS) data versus improved observations from the Ozone Monitoring Instrument (OMI). Atmospheric Environment, 110, 130-143. Recuperado de https://www.sciencedirect.com/science/article/pii/S1352231 015002794

Lasswell, H. (1956). The decision process: Seven categories of functional analysis. College Park: Universidad de Maryland.

Lasswell, H. (1971). A pre-view of policy sciences. Nueva York: American Elsevier Publishing

Levy, J., Wilson, A. y Zwack, L. (2007). Quantifying the efficiency and equity implications of power plant air pollution control strategies in the United States. Environmental Health Perspectives, 115(5), 743-750. Recuperado de https:/www.ncbi.nlm.nih.gov/pmc/articles/PMC 1867973/

Ma, Y., Richards, M., Ghanem, M., Guo, Y. y Hassard, J. (2008). Air pollution monitoring and mining based on sensor grid in London. Sensors, 8(6), 3601-3623. Recuperado de http://www.mdpi.com/1424-8220/ $8 / 6 / 3601 / \mathrm{htm}$

Mickwitz, P. (2003). A framework for evaluating environmental policy instruments: Context and key concepts. Evaluation, 9(4), 415-436. Recuperado de http://journals.sagepub.com/doi/pdf/10.1177/13563890 03094004

Miranda, A., Silveira, C., Ferreira, J., Monteiro, A., Lopes, D., Relvas, ... Roebeling, P. (2015). Current air quality plans in Europe designed to support air quality management policies. Atmospheric Pollution Research, 6(3), 434-443. Recuperado de https://www.sciencedirect.com/ science/article/pii/S1309104215302129

Naiker, Y., Diab, R., Zunckel, M. y Hayes, E. (2012). Introduction of local air quality management in South Africa: Overview and challenges. Environmental Science and Policy, 17, 62-71. Recuperado de https:// www.sciencedirect.com/science/article/pii/S1462901111001808

Organización Mundial de la Salud (OMS). (2014). 7 million premature deaths annually linked to air pollution. Ginebra, Suiza. Recuperado de http:// www.who.int/mediacentre/news/releases/2014/air-pollution/en/ 
O'Toole, J. L. (2004). The theory-practice issue in policy implementation research. Public Administration, 82(2), 309-329. Recuperado de https:// onlinelibrary.wiley.com/doi/epdf/10.1111/j.0033-3298.2004.00396.x

Perevochtchikova, M. (2009). La situación actual del sistema de monitoreo ambiental en la Zona Metropolitana de la Ciudad de México. Estudios Demográficos y Urbanos, 24(3), 513-547. Recuperado de http://estudios demograficosyurbanos.colmex.mx/index.php/edu/article/view/1327/ 1320

Secretaría del Medio Ambiente de la Ciudad de México (SMA). (2016a). Inventario de emisiones de la CDMX 2014. Ciudad de México: Dirección General de Gestión de la Calidad del Aire.

Secretaría del Medio Ambiente de la Ciudad de México (SMA). (2016b). Calidad del aire en la Ciudad de México. Informe 2015. Ciudad de México: Dirección General de Gestión de la Calidad del Aire.

Sivertsen, B. (2008). Monitoring air quality, objectives and design. Chemical Industry and Chemical Engineering Quarterly, 14(3), 167-171. Recuperado de http://www.ache.org.rs/CICEQ/2008/No3/03_14\%283\% 29_2008.pdf

Stavins, R. (2003). Experience with market-based environmental policy instruments. En D. Bromley (ed.), Handbook of environmental economics (pp. 355-435). Nueva York: Elsevier.

Touma, J., Isakov, V., Ching, J. y Seigneur, C. (2006). Air quality modeling of hazardous pollutants: Current status and future directions. Journal of the Air and Waste Management Association, 56(5), 547-558. Recuperado de http://citeseerx.ist.psu.edu/viewdoc/download?doi=10.1.1.174.3 070\&rep $=$ rep $1 \&$ type $=$ pdf

Wang, X., Hawkins, C., Lebredo, N. y Berman, E. M. (2012). Capacity to sustain sustainability: A study of US cities. Public Administration Review, 72(6), 841-853. Recuperado de https://www.researchgate.net/publication/264740184_Capacity_to_Sustain_Sustainability_A_Study_of_US Cities

Wolfram, M. (2016). Conceptualizing urban transformative capacity: A framework for research and policy. Cities, 51, 121-130. Recuperado de https://www.sciencedirect.com/science/article/pii/S0264275115300032 Zuk, M., Tzintzun, G. y Rojas, L. (2007). Tercer almanaque de datos y tendencias de la calidad del aire en nueve ciudades mexicanas. Ciudad de México: INE / Semarnat. 


\section{Acerca del autor}

Alejandro Navarro Arredondo es doctor en Ciencias Políticas y Sociales por la Universidad Nacional Autónoma de México (UNAM), maestro en Administración y Políticas Públicas por el Centro de Investigación y Docencia Económicas (CIDE) e investigador visitante en la Escuela de Asuntos Públicos de la Universidad de Texas. Se ha desempeñado como investigador del Instituto Belisario Domínguez de la Cámara de Senadores, en proyectos sobre finanzas públicas, desarrollo local, participación ciudadana, educación y política social. Actualmente es profesor-investigador de la Universidad Autónoma Metropolitana, Unidad Xochimilco, División de Ciencias Sociales y Humanidades, Departamento de Política y Cultura. Es miembro del SNI, nivel I.

Algunas de sus publicaciones más recientes son:

Navarro Arredondo, A. (2015). Deuda en estados y municipios en México. En V. Peña y C. González (coords.). Deudas en estados y municipios. Aproximaciones a una problemática subnacional. Hermosillo, México: El Colegio de Sonora / Centro de Investigación Económica y Presupuestaria.

Navarro Arredondo, A. (2014). Cooperación internacional para la protección ambiental en municipios mexicanos. Políticas Públicas, 2(1), pp. 23-33.

Recepción: 27 de septiembre de 2017.

Aceptación: 13 de abril de 2018. 
